\title{
Integración metodológica como herramienta de investigación para las relaciones internacionales
}

\author{
Methodological integration as a research tool for international relations
}

A integração metodológica como ferramenta de pesquisa para as relações internacionais

Kenia María Ramírez Meda

Universidad Autónoma de Baja California, Facultad de Ciencias Sociales y Políticas,

México

kenia@uabc.edu.mx https://orcid.org/0000-0001-8813-4422

Fernando David Márquez Duarte University of California, United States of America fdmdj@hotmail.com https://orcid.org/0000-0001-5653-5002

\section{Resumen}

Este artículo tiene el propósito de aproximar al lector a la aplicación de los métodos para el estudio de la ciencia de las Relaciones Internacionales, con especial énfasis en la integración metodológica. Con esto en mente, se procederá primeramente a explicar en qué consiste la metodología cualitativa, en qué se diferencia de la metodología cuantitativa y cuáles son sus herramientas más utilizadas. En un segundo apartado, se adentra al lector a la utilización del método comparativo, el cual, brinda resultados que permiten explicar patrones y regularidades entre distintos sistemas sociales, políticos e institucionales. En un tercer momento se aborda la integración metodológica, resultado de la utilización de diferentes métodos, al integrar la información cualitativa a cuantitativa, con el objetivo final de que el análisis y procesamiento de la información sea más completo y esto lleve a conclusiones más certeras e integrales. Enseguida, se ilustra la aplicación de la integración metodológica con una investigación realizada desde la perspectiva de estudio de caso comparativo en la frontera de México y Estados Unidos. Finalmente se presentan las conclusiones del trabajo. 
Palabras clave: integración metodológica, método comparativo, métodos cualitativos, relaciones internacionales.

\section{Abstract}

This article is intended to bring the reader to the application of methods in the study of International Relations science with special emphasis on methodological integration. With this in mind, first we explain the qualitative methodology, and how it differs from the quantitative methodology and which are their tools. In a second part, we introduce to the reader to the use of the comparative method, which provides extremely rich results since it allows to explain patterns and regularities between different social, political and institutional systems. In a third part, we address the methodological integration, as a result of the use of different methods, to integrate qualitative with quantitative data, with the ultimate goal of a more complete analysis and processing of information which leads to more accurate and comprehensive conclusion. Afterwards, we illustrate the application of the methodological integration with a research carried out from the perspective of comparative case study on the border between Mexico and the United States. Finally, we present the conclusions of the work.

Keywords: methodological integration, comparative method, qualitative methods, international relations.

\section{Resumo}

Este artigo tem por objetivo aproximar o leitor da aplicação dos métodos para o estudo da ciência das Relações Internacionais, com especial ênfase na integração metodológica. Com isso em mente, iremos primeiro explicar em que consiste a metodologia qualitativa, como ela difere da metodologia quantitativa e quais são as suas ferramentas mais utilizadas. Em uma segunda seção, o leitor é apresentado ao uso do método comparativo, que fornece resultados que permitem explicar padrões e regularidades entre diferentes sistemas sociais, políticos e institucionais. Num terceiro momento, aborda-se a integração metodológica, resultado da utilização de diferentes métodos, integrando informação qualitativa à quantitativa, com o objetivo final de que a análise e processamento da informação seja mais completo e isso conduza a conclusões integral. A seguir, a aplicação da integração metodológica é ilustrada com uma investigação realizada na perspectiva de um estudo de caso comparativo na fronteira do México com os Estados Unidos. Por fim, são apresentadas as conclusões do trabalho. 
Palavras-chave: integração metodológica, método comparativo, métodos qualitativos, relações internacionais.

\section{Introducción}

Desde sus inicios, el estudio de las relaciones internacionales (RR. II.) ha estado acompañado de un intenso debate teórico y metodológico que aún sigue vigente. Por ejemplo, según la opinión de algunos internacionalistas, la falta de consenso en torno a un solo método de investigación para dicha área se ha traducido en un obstáculo para su avance científico, mientras que otros sostienen que es justamente la existencia de numerosos métodos y enfoques, junto con el debate que provocan, lo que ha facilitado el progreso de las aportaciones teóricas en un campo de estudio marcado por su complejidad y dinamismo. Sin embargo, a pesar de estas posturas encontradas, en la actualidad el surgimiento de métodos y técnicas de investigación novedosas en las RR. II. han facilitado a los estudiosos de esta disciplina el logro de indagaciones con mayor rigor científico y profundidad. Por eso, se ha explorado la posibilidad de entrelazar técnicas de investigación de ambas corrientes (cualitativas y cuantitativas) para procurar un análisis más completo de este objeto de estudio.

Al respecto, Monje (2011) señala: “A pesar de que cada opción metodológica se sustenta en supuestos diferentes y tiene sus reglas y formas básicas de acción, establecidas y compartidas por la propia comunidad científica, no son métodos excluyentes, [pues] se complementan" ( p. 11).

La estructura del artículo es la siguiente: primero se explica en qué consiste la metodología cualitativa, cuáles son sus diferencias con la metodología cuantitativa y cuáles herramientas de este método son las más utilizadas. En segundo lugar, se expone la utilización del método comparativo, el cual brinda elementos que permiten explicar patrones y regularidades entre distintos sistemas sociales, políticos e institucionales. En tercer lugar, se aborda la integración metodológica de información cualitativa y cuantitativa con el objeto de que el análisis y el procesamiento de los datos sean más completos, lo que sirve para generar conclusiones más certeras e integrales. Luego se ilustra la aplicación de estos métodos con los resultados de una investigación realizada desde la perspectiva de estudio de caso comparativo en la frontera de México y Estados Unidos. Finalmente, se presentan las conclusiones. 


\section{Una aproximación a la metodología cualitativa aplicada al estudio de las relaciones internacionales}

Los métodos cualitativos utilizados en la investigación de las RR. II. no son exclusivos de una posición epistemológica en particular. La metodología cualitativa hace referencia a la recolección de datos y estrategias de análisis de datos no numéricos con el objeto de brindar un mejor entendimiento sobre cómo se percibe el mundo, por lo que exige enfocarse en los significados y en los procesos que constituyen a las RR. II. Esto se logra a través de un análisis profundo de eventos, fenómenos, países, regiones, organizaciones o individuos (Creswell, 2003; Lamont, 2015).

La metodología cualitativa ofrece datos descriptivos y/o de relaciones causales del comportamiento humano en todas sus manifestaciones (escritas, habladas $\mathrm{u}$ observadas), y se caracteriza por estudiar en detalle situaciones concretas. Su fin último es trascender la causalidad del objeto de estudio para llegar a su comprensión (HernándezSampieri, Fernández y Baptista, 2010). Frecuentemente, este tipo de métodos depende del razonamiento inductivo, pues sigue una secuencia lógica a partir de la observación de los fenómenos o hechos de la realidad, es decir, desde observaciones empíricas se llega a la construcción de proposiciones teóricas.

Hernández-Sampieri et al. (2010) sugieren que, desde la metodología cualitativa, el planteamiento del problema que se va investigar debe iniciar con la definición del concepto principal, seguido de una constante revisión de la propuesta durante la recolección de datos. Es decir, a lo largo del proceso se generará la hipótesis, la cual será redefinida a partir de la obtención de datos, aunque también puede ser producto del estudio (en ambos casos no será probada estadísticamente). Por ello, Collier (2011) considera que estas hipótesis son emergentes, flexibles y contextuales, pues se adaptan a los datos y al entorno de la investigación. Por ejemplo, en la investigación cualitativa al usar un enfoque de teoría enraizada - se acude primero al campo y luego, a partir de ese trabajo exploratorio, se construyen las hipótesis y los instrumentos metodológicos. En otras palabras, con este procedimiento primero se explora el caso y luego se construyen las hipótesis, para lo que se utiliza el método de process tracing.

A diferencia de los métodos cuantitativos en las RR. II., el tamaño de la muestra de la investigación no es representativo del universo a estudiar, ya que el fin último de quien investiga es profundizar en su objeto de estudio. Así, la muestra puede estar conformada por individuos, sucesos y/o documentos. En este sentido, tiene mayor peso la calidad de la muestra que la cantidad. Pero ¿cómo definir el tamaño de esta? Dependerá del interés en el caso, de las limitaciones del investigador para el manejo de una cierta 
cantidad de sucesos o fenómenos, del número de hechos que se pueden responder a partir de la pregunta de investigación y de si los casos elegidos son accesibles y frecuentes para el investigador.

Es importante resaltar que la definición de muestra en este tipo de metodología puede ser modificada en la medida que el estudio progresa y suele ser cualitativamente significativa, a diferencia de lo que sucede en los estudios cuantitativos, donde es estadísticamente significativa. En la tabla 1 se observan las principales diferencias entre la investigación cualitativa y la cuantitativa.

Tabla 1. Características de la investigación cualitativa y cuantitativa

\begin{tabular}{|c|c|c|}
\hline Comparación & Investigación cualitativa & Investigación cuantitativa \\
\hline Centro de interés & Cualidad. & Cantidad. \\
\hline Raíces filosóficas & $\begin{array}{l}\text { Fenomenología, } \\
\text { interacción simbólica. }\end{array}$ & $\begin{array}{l}\text { Positivismo, empirismo } \\
\text { lógico. }\end{array}$ \\
\hline Conceptos asociados & $\begin{array}{l}\text { Trabajo de campo, } \\
\text { etnografía, subjetividad. }\end{array}$ & $\begin{array}{l}\text { Experimental, empírica, } \\
\text { estadística. }\end{array}$ \\
\hline Objetivos de investigación & $\begin{array}{l}\text { Comprensión, descripción, } \\
\text { descubrimiento, } \\
\text { generadora de hipótesis. }\end{array}$ & $\begin{array}{l}\text { Predicción, control, } \\
\text { descripción, confirmación, } \\
\text { comprobación de hipótesis. }\end{array}$ \\
\hline Características del diseño & $\begin{array}{l}\text { Flexible, envolvente, } \\
\text { emergente. }\end{array}$ & $\begin{array}{l}\text { Predeterminado, } \\
\text { estructurado. }\end{array}$ \\
\hline Escenario & Natural, familiar. & Desconocido, artificial. \\
\hline Muestra & $\begin{array}{l}\text { Pequeña, no aleatoria, } \\
\text { teórica. }\end{array}$ & $\begin{array}{l}\text { Grande, aleatoria, } \\
\text { representativa. }\end{array}$ \\
\hline Compilación de datos & $\begin{array}{l}\text { Entrevistas, observaciones, } \\
\text { papel del investigador. }\end{array}$ & $\begin{array}{l}\text { Instrumentos inanimados } \\
\text { (escalas, } \\
\text { encuestas, cuestionarios). }\end{array}$ \\
\hline Modalidad de análisis & Inductivo. & $\begin{array}{l}\text { Deductivo (uso de las } \\
\text { estadísticas). }\end{array}$ \\
\hline
\end{tabular}

Fuente: Elaboración propia con base en Hernández-Sampieri et al. (2010)

En cuanto a los instrumentos para la obtención de datos cualitativos, se destacan la observación, la entrevista, los grupos focales y la investigación de gabinete. Sin embargo, Lamont (2015) explica que los datos cualitativos también se encuentran en formas no textuales de expresión, como monumentos, mapas, arte, construcciones sociales e incluso herramientas visuales. Por ello, este autor señala que los métodos para la recolección de datos cualitativos utilizados por los estudiosos de las RR. II. han sido particularmente los siguientes:

- Entrevistas. Se trata de una herramienta que permite recopilar información a fondo. Los investigadores recurren a las entrevistas para encontrar datos sobre un fenómeno en particular, un evento o un objeto, o para descubrir las opiniones de 
una persona o un grupo de personas. Las entrevistas pueden ser estructuradas, en las cuales se aborda al entrevistado con preguntas previamente escritas y con categorías de respuestas; asimismo, pueden ser semiestructuradas, en las cuales se tiene cierto grado de flexibilidad, aunque el investigador continúa guiándose por una estructura básica de entrevista. Además, existen entrevistas sin estructura, las cuales permiten una conversación fluida con el entrevistado en un diálogo abierto, aunque por su misma naturaleza es deseable que sea registrada en audio o video. Finalmente, las entrevistas pueden ser semidirectivas, donde se le da libertad al entrevistado para que relate todo lo que desee; aquí el entrevistador solo guía la discusión sin interrumpir al entrevistado.

- Focus groups. Generalmente se trata de reunir un grupo de entre seis y diez participantes para discutir un tema y una serie de preguntas. El objetivo es recabar información sobre cómo se percibe cierto tema en un contexto de interacción social. Todos los focus groups son conducidos por un moderador. Al respecto, Hamui y Varela (2013) señalan: "Una de las figuras centrales en un grupo focal es el moderador, quien dirige el diálogo basado en la guía de entrevista, previamente elaborada, da la palabra a los participantes y estimula su participación equitativa" (p.57).

- Búsqueda basada en internet. Si bien el internet tiene muchas ventajas prácticas, cuando no se consultan materiales académicos y científicos hay que ser muy cautelosos en cuanto a la veracidad de la información presentada. Con este criterio, internet permite acceder a medios de comunicación de todo el mundo, sitios web gubernamentales y páginas oficiales de prácticamente todos los organismos internacionales. En palabras de Lorenzo

- Domínguez y Xalabarder (1997), "los investigadores pueden hallar en Internet una herramienta útil para sus trabajos a partir de dos aspectos fundamentales: la capacidad de comunicación que ofrece la red, y el enorme volumen de información que día tras día se asoma en el ciberespacio" (p. 1)

- Búsqueda documental y de archivo. También conocida como investigación de gabinete, es una herramienta sumamente valiosa para investigar sobre un conflicto internacional, una organización internacional, políticas públicas, legislaciones, entre otros. Hay que tomar en cuenta que se pueden dar de dos tipos: las fuentes primarias (son originales y el investigador tiene acceso a ellas de primera mano) y las fuentes secundarias (obtenidas mediante el análisis de las fuentes primarias). La limitación de esta herramienta es que en muchos casos, dada la naturaleza del 


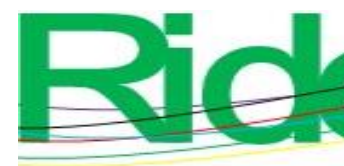

Revista Iberoamericana para la Investigación y el Desarrollo Educativo ISSN $2007-7467$

campo de estudio de las RR. II., el investigador no tiene acceso a los materiales confidenciales de un gobierno u organización, de ahí que sea necesario recabar información por otros medios. Esto, no obstante, ofrece una desventaja crucial, pues provoca que solo se pueda reinterpretar la información original. En este sentido, se reconoce que muchos datos son ocultados por los gobiernos, organizaciones y empresas "por razones de seguridad, por temor a una reacción negativa de la opinión pública, o de la oposición política. Existe un mecanismo de autocensura en los documentos escritos, por lo que estos solo reflejan parcialmente los procesos institucionales internos" (Salazar, 2004, p. 200). Recopilada la información cualitativa, el investigador se enfrentará a la cuestión de cómo analizarla, lo que se puede prestar para generar interpretaciones erróneas. Aun así, algunas de las técnicas de análisis más usadas cuando se recaba información cualitativa son las siguientes:

- El análisis de contenido. En ocasiones se le considera una herramienta de método cuantitativo, pues busca convertir los datos textuales en forma numérica. El objetivo es analizar grandes cantidades de información a través de la codificación o la categorización. Es una técnica mixta de investigación (cualitativa y cuantitativa) que puede incluir datos escritos, fotografías, programas de televisión, películas y otras expresiones artísticas. Una vez que se pasa a la codificación de los datos a categorías, es posible ubicar la presencia de ciertas tendencias o patrones. El fin último del análisis de contenido es encontrar elementos particulares en la comunicación (Lamont, 2015).

- El análisis del discurso. Es un análisis cualitativo que se enfoca en la interpretación de manifestaciones lingüísticas orales o escritas, y puede incluir formas oficiales y no oficiales de comunicación. Se centra en conocer cómo y por qué ciertas retóricas emergen, se vuelven dominantes y son utilizadas por los actores bajo estudio. Para aplicar este tipo de análisis es necesario identificar y justificar la selección de textos a estudiar, independientemente del tipo de documento, discurso o declaración que se defina. Aun así, debe tratarse de textos que provengan de fuentes autorizadas, bien informadas y con dominio del tema a estudiar. Se trata de discernir el cómo y por qué los actores recurrieron a esa retórica en particular, pues se procura descubrir cómo el lenguaje preconfigura el mundo en que vivimos; de hecho, también se pueden hacer análisis de las 
cantidades de veces que se usan ciertas palabras o de sus relaciones en un discurso (Lamont, 2015).

Por otra parte, también cabe recomendar la obra de Klotz y Prakash (2008), quienes desentrañan el diseño de investigación cualitativo para señalar algunas de las estrategias más usadas, las cuales se describen a continuación:

- Análisis del discurso. Se estima que para entender el discurso se deben seguir cuatro pasos metodológicos. Primeramente, es de suma importancia poseer un grado de competencia cultural para adentrarse correctamente en la manera en que se ve el mundo. A partir de ahí, se ofrece una guía para la delimitación de textos y el mapeo posterior de las representaciones que comprenden el discurso. En último lugar, se busca esclarecer la estratificación de los discursos dominantes y subordinados en las RR. II. (Klotz y Prakash, 2008).

- Representaciones históricas. El enfoque en la narrativa de la historia diplomática ha prevalecido en el estudio cualitativo de las RR. II., por lo que es importante tener herramientas de análisis histórico, así como guías para la búsqueda de materiales de archivo y para delimitar la selección de material. Por ello, se puede afirmar que la historia es una ciencia fundamental para el estudio de las RR. II. $\mathrm{Al}$ respecto, Rojas (2004) señala:

El mayor aporte de la historia a las RI consiste en tratarlas como un objeto histórico, en examinar su discurso como resultado de una conjunción de factores en un momento específico, en correr el velo de la reificación que los teóricos de lo internacional han querido mantener; la historia, lo que nos evidencia, es la propia historicidad de las RI (p.3).

- Investigación etnográfica. Contrario a la historiografía, la etnografía es utilizada poco como herramienta de análisis en las RR. II. Sin embargo, en tiempos recientes, dicha visión ha cambiado, pues se considera que la observación y la entrevista participantes pueden ayudar a responder preguntas relacionadas con los estudios internacionales. Hurtado (2016) lo explica del siguiente modo:

Este método tiene la capacidad de volver palpable y visible a las personas “comunes", ajenas al ámbito académico, la materialidad de las prácticas sociales que ocurren al interior de las instituciones estatales, en zonas de guerra o refugiados, en lugares de participación de la sociedad civil que se mueve en el ámbito internacional, en organismos internacionales, y en los ámbitos 


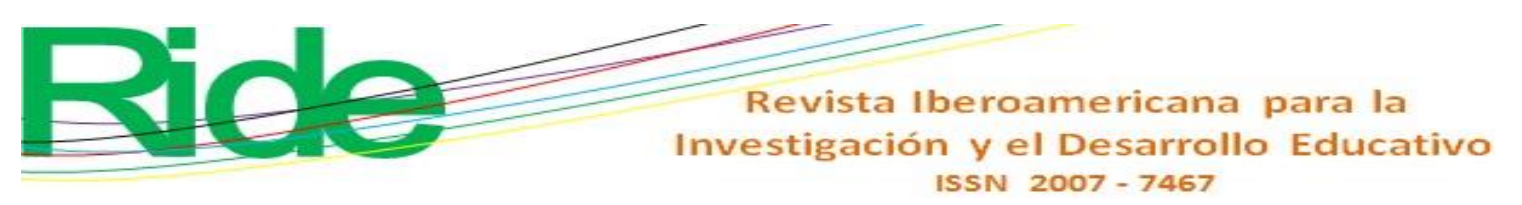

académicos que suelen verse como externos al objeto de estudio (p. 2).

- Seguimiento de procesos. Comúnmente conocido como process tracing, es usado en el análisis cualitativo en RR. II. para enfocarse en individuos como actores clave y para el seguimiento del proceso al ser vinculado con el estudio de los mecanismos causales en general y entendimientos de casos y procesos complejos a lo largo del tiempo, donde se busca crear espacios rivales para someter a prueba hipótesis contrastantes (Zaks, 2017).

En síntesis, la metodología cualitativa puede brindarnos un mejor entendimiento de cómo se percibe al mundo. Para cumplir con dicho cometido se deben analizar los significados y los procesos que construyen a las RR. II., para lo cual se pueden emplear las herramientas mencionadas en este apartado.

\section{Objetivos}

- El propósito de la presente investigación es demostrar que un enfoque mixto puede servir para interpretar de forma más precisa la participación ciudadana juvenil en Baja California (México) y California (Estados Unidos) para lo cual se han planteado los siguientes objetivos específicos:

- Aplicar el método comparado (MC) utilizando el tema participación ciudadana juvenil en Baja California (México) y California (Estados Unidos): el caso de los Modelos de Naciones Unidas (MUN) como ejemplo de la integración metodológica en fenómenos de las RR. II.

- Reflexionar sobre la aplicación de la metodología de integración metodológica en fenómenos y sucesos de las RR. II.

Antes de describir la metodología y señalar los resultados, vale acotar que los Modelos de Naciones Unidas (MUN) se definen como una actividad educativo-cultural que intenta representar el protocolo de la Organización de Naciones Unidas (ONU) a través de un "juego de rol" que reproduce el modo en que se reúnen y negocian los distintos países del mundo sobre temas de interés para la humanidad. En el MUN "los alumnos representan a los delegados de diferentes países e intervienen en debates y negociaciones en los que tratan temas que corresponden a los programas de trabajo de los diferentes órganos y comisiones de las Naciones Unidas" (Centro de Información de la ONU para México, Cuba y República Dominicana [CINU], s. f., párr.3). Estos MUN son instrumentos prácticos que replican las actividades de los principales comités de las Naciones Unidas y de otros organismos internacionales, y se pueden ejecutar en todos los 


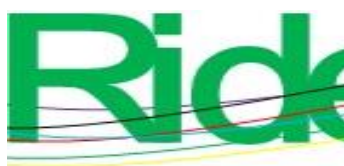

Revista Iberoamericana para la Investigación y el Desarrollo Educativo ISSN $2007-7467$

niveles educativos. En este sentido, los estudiantes debaten, discuten y plantean propuestas de solución a las temáticas de la agenda internacional designadas por el comité organizador. El objetivo es ofrecer una postura según el país al que representan, apegándose a los principios de política exterior de esa nación y del protocolo del OI simulado.

Los MUN existen prácticamente desde la fundación de la ONU. De hecho, se documenta que el primer ejercicio de esta naturaleza se llevó a cabo en 1947. Para Obendorf y Randerson (2012) "los MUN son hoy un fenómeno global, entregado en casi todos los niveles de la enseñanza y el aprendizaje, desde las escuelas primarias y secundarias y universidades a través de pregrado y de postgrado en universidades" (p. $351)$.

Por ello, la pregunta formulada en el presente estudio fue si estos ejercicios fomentan la participación ciudadana juvenil. Al respecto, se argumenta que los MUN son herramientas formativas, en especial, en los niveles de educación superior, dado que propician habilidades en sus participantes, tales como expresión oral y escrita, búsqueda de información, redacción y debate. Además, promueve valores como la tolerancia, la comprensión intercultural, la empatía, entre otros. Esto resulta particularmente importante para los estudiantes de disciplinas como las RR. II., puesto que deben comprender la cambiante y compleja realidad internacional que se desarrolla en medio de situaciones turbulentas (Zenuk-Nishide, 2014).

\section{Metodología}

La presente indagación se sustentó en integración metodológica , la cual se aplicó con base en un enfoque de estudio de caso comparativo (Creswell, 2003). En este enfoque se usan múltiples fuentes de información (p. ej., entrevistas, observaciones, revisión bibliográfica, etc.) y se analiza, especialmente, el contexto de los casos.

En esta investigación se contó con una muestra cualitativamente significativa, pues se entrevistaron a unidades de información relevantes para los casos. En concreto, dichas unidades fueron jóvenes que han participado en MUN en Tijuana y en Mexicali (México), así como en San Diego (Estados Unidos). El principio de homogeneidad de los entrevistados fue claramente que habían participado en MUN, mientras que el principio de heterogeneidad se manifiesta aspectos diferenciadores, como la escolaridad (estudiantes y egresados de la universidad), el sexo, el rol que ejercieron en los MUN y su ubicación (estados de diferentes países). El trabajo de campo, en síntesis, permitió recabar información mediante un total de 18 entrevistas que se aplicaron del siguiente 
modo: 11 realizadas a participantes de MUN de Tijuana y Mexicali (Baja California, México) y 7 a participantes de MUN de San Diego (California, Estado Unidos). El instrumento empleado estaba conformado por los siguientes apartados:

- Datos generales (nombre, sexo, escolaridad, datos socioeconómicos).

- Datos generales de participación en MUN (cantidad de veces que ha participado, en dónde ha participado, qué papel ha jugado, nacionalidad).

- Dimensión social (experiencias de participación social previas a MUN, medición por dimensiones de la participación social antes de participar en MUN).

- Dimensión directa (medición dicotómica de los indicadores de participación ciudadana juvenil).

- Dimensión electoral-política (medición dicotómica de los indicadores de participación electoral-política).

- Capacidades y formación ciudadana (adquisición de habilidades, aptitudes, conocimientos).

A través de esta metodología se pretende analizar si los modelos de naciones unidas (MUN) fomentan la participación ciudadana juvenil. Las entrevistas (semiestructuradas y semidirectivas) se realizaron de forma presencial y a través de videollamadas entre los años 2017 y 2018.

Los datos fueron procesados mediante el software Atlas ti. Luego, la información codificada se agrupó en diferentes categorías: social, directa y electoral-política. Asimismo, cabe resaltar que el análisis y la codificación se separaron en los MUN de California y los de Baja California, los cuales luego fueron comparados.

Después de esta codificación cualitativa se realizó una codificación cuantitativa, pues también se usaron las escalas de Likert-Thurstone, así como la dicotómica. En otras palabras, se codificaron los indicadores para efectuar la integración metodológica y medir el grado de participación ciudadana juvenil. Esto se concretó usando la escalera de participación juvenil de Hart (2013) y el espectro de participación pública de Stuart (2017).

Las preguntas con opciones de respuesta dicotómicas y de tipo Likert-Thurstone se formularon con el objetivo de medir la intensidad en cada uno de los indicadores considerados. Con las preguntas dicotómicas (sí o no) se determinó si los jóvenes habían participado, por ejemplo, en un referendo, acción individual no-continua donde no se puede medir intensidad.

El uso de la escala de tipo Likert-Thurstone es más complejo porque resulta de la combinación de dos estrategias de medición, de ahí que primero se deba determinar su 
función. En la escala Likert hay un rango de opciones de respuesta más variado (mayor a dos), por lo que es utilizada ampliamente en estudios de mercado o de satisfacción del cliente, ya que es posible tener un rango amplio de opciones. Por ejemplo, para la pregunta ¿Qué tan probable es que recomiende nuestros servicios a sus conocidos?, las opciones podrían ser "muy probable", "probable", "indiferente”, "poco probable", "no lo recomendaría". Cada una de estas opciones se codifica con un número con el objetivo de facilitar el análisis de los resultados del instrumento. De esta manera se comienza la integración metodológica al otorgar un valor numérico a diferentes opciones de respuesta cualitativas.

En cambio, la escala de tipo Thurstone es aquella en la que la persona que diseña el instrumento le asigna valores predeterminados a cada pregunta, de manera que algunas tienen mayor valor que otras. Esto se realiza con el fin de tener una medición más adecuada, como sucede en la presente investigación, donde ciertos indicadores, según la literatura, tienen mayor peso que otros debido a su importancia o a sus implicaciones.

Al utilizar los métodos de estos dos tipos de escalas se le nombra de tipo LikertThurstone, pues las preguntas tienen un rango de respuesta mayor a dos opciones y se les pueden asignar valores predeterminados diferenciados según cada indicador (Bozal, 2006).

\section{Implementación del método comparativo en estudios internacionales}

La comparación es siempre útil para alcanzar el objetivo de una investigación, pues permite identificar semejanzas y diferencias, y otorga la posibilidad de controlar variables. Para Nohlen, Schultze y Romano (2006), este método busca examinar nexos causales y trata de aislar los factores que pueden ser considerados como de causa o de efecto. Además, resulta útil para distinguir entre el modo de utilización cuantitativo y cualitativo, ya que se refiere al número de casos que se van a analizar y a la racionalidad con que se guía el análisis. Entre las principales formas de usar el método comparativo se encuentran el método de diferencia y el de similitudes de John Stuart Mill (Savolainen, 1994).

El método comparativo (MC) se considera una especialización del método científico, y se puede concretar en diferentes tipos: experimental, estadístico, histórico y comparación de atributos compartidos y no compartidos. La investigación comparativa 


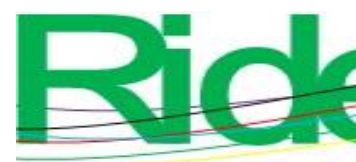

Revista Iberoamericana para la Investigación y el Desarrollo Educativo ISSN $2007-7467$

generaliza más, mientras que el estudio de caso resulta más individualista, por lo que ambos se refuerzan entre sí.

En las ciencias sociales existen distintas formas de explicar y comparar, aunque específicamente se pueden mencionar tres tipos de trabajos científicos: los ideográficos, que son descriptivos e interpretativos; los teóricos, cuyo fin es hacer progresar la teoría, y los comparatistas, que se ubican en una fase intermedia de los dos anteriores. Estos últimos cuentan con menos seguidores; sin embargo, en los últimos años los estudios comparativos han ganado una importante atención como metodología de estudio en las ciencias sociales y como un método para lograr el control de variables.

Para realizar un óptimo proceso del método comparativo el aspecto conceptual resulta de fundamental importancia, así como la clasificación de casos, conceptos y variables, es decir, la operacionalización. En pocas palabras, la estructura teórica resulta fundamental, así como la formulación de hipótesis, la cual se apoya en estudios precedentes.

Dentro del MC en las RR. II. resulta usual el estudio de casos comparativos. En este tipo la investigación suele ser de base cualitativa, y se emplean múltiples fuentes de información, como entrevistas, observaciones, revisión bibliográfica y hemerográfica, etc., pero especialmente se analiza ampliamente el contexto de los casos. Los siguientes son algunos de los aspectos que se deben considerar al momento de aplicar el MC en los estudios internacionales:

- Espacio: Aumentar el número de casos implica elevar la cantidad de variables relevantes, lo que hace más difícil la comparación; sin embargo, también se puede elegir un solo caso y comparar hipótesis con la literatura existente o en diferentes periodos. Si se utilizan dos o más casos, sería un estudio de tipo binario. Es recomendable, entonces, utilizar pocos casos y muchas variables (siempre menos de 20 casos). Pero ¿cómo elegir los casos? Para ello, se deben considerar factores comunes y diferentes.

- Para Lijphart (1971) debe existir un número reducido de casos (entre 2 y menos de 20), ya que el MC se utiliza para investigaciones con recursos modestos; por tanto, reducir el número de variables basándose en su contexto y en la teoría es lo más recomendable. Dentro del tipo de estudios de caso comparativos existe un subtipo "clínico", donde se analizan casos específicos en cantidad limitada de una situación acotada. El propósito es capturar a "todo" el individuo de los casos de manera intensiva. Este tipo de investigación también se puede entender como 
configurativo-ideográfico, y se usa para analizar a individuos de colectivos complejos. La validez recae en el estudio intensivo de los casos (Gomm, Hammersley y Foster, 2000).

- Tiempo: El análisis comparativo puede realizarse en diferentes momentos (comparación diacrónica) o en momentos sincrónicos.

- Variables: Se recomienda la reducción de variables, pero solo acudiendo a teorías e hipótesis previamente establecidas. La utilización de variables clave es necesaria.

Las investigaciones con $\mathrm{N}$ (muestra) pequeñas (pocos casos) son recomendables para el método comparativo. En tal sentido, la técnica de "muchas variables, pocos casos" permite lograr analogías profundas. Por otro lado, existen técnicas cuantitativas para investigaciones de muchos casos que pueden ayudar a procesar la información y complementar las conclusiones en el MC; por ejemplo, las estrategias de muestreo, significación estadística, regresión, varianza, análisis multivariante, entre otros.

Los resultados del MC permiten explicar patrones y regularidades entre distintos sistemas sociales, políticos e institucionales, así como identificar las variables independientes que pueden explicar las dependientes (resultados). Nuevamente, se enfatiza la importancia de la teoría y la construcción de las hipótesis como aspectos fundamentales del MC. Asimismo, en el caso de los estudios internacionales estas deben ser aplicables a más de un país o cultura y permitir la utilización de indicadores similares.

\section{La integración metodológica}

La integración metodológica cualitativa es una herramienta útil en la investigación de las ciencias sociales - especialmente en estudios de RR. II. y en ciencia política (CP) — debido a que a lo largo de los años estas disciplinas han sido criticadas por no tener métodos probables para basar teorías, hipótesis y propuestas conceptuales. Anteriormente, en el caso de las RR. II., las teorías se basaban en inferencias teóricas y en indicadores abstractos que eran imposibles de medir, como la naturaleza humana o el análisis del comportamiento de individuos específicos (un claro ejemplo es el behaviorismo). Con este instrumento, diferentes ciencias sociales han logrado responder a estas críticas, dotándole de mayor credibilidad y legitimidad a las investigaciones desarrolladas.

La integración metodológica es un método que se basa en un análisis cualitativo y cuantitativo de cualquier información recabada (Patterson, 1996). Esta puede ser usada 


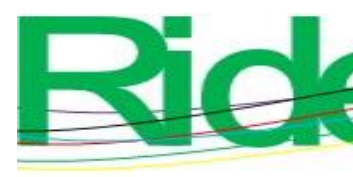

Revista Iberoamericana para la Investigación y el Desarrollo Educativo ISSN $2007-7467$

para examinar diferentes técnicas de investigación cualitativa, de manera que sea procesada y traducida en información cuantitativa para que logre ser medible de una manera más precisa. De este modo se procura conseguir conclusiones más certeras e integrales.

La aplicación de la integración metodológica cualitativa en RR. II. y CP se concreta principalmente por medio del procesamiento cuantitativo de la información cualitativa recabada, por ejemplo, mediante una entrevista. Sin embargo, vale resaltar que cuando se usa la integración metodológica de información cualitativa a cuantitativa, la racionalidad de la investigación se mantiene cualitativa, pues solo se usa la integración para traducir cierta información recabada a datos numéricos.

Una aplicación concreta de la integración metodológica puede ser la medición de algún aspecto o conjunto de aspectos; no obstante, es importante considerar que para tener un análisis útil mediante la integración es necesario que la estructura metodológica de una investigación sea sólida, ya que es importante saber — antes de recabar y procesar la información - para qué se utilizará la integración, de manera que no sea solo un instrumento sin un fin. Una parte crucial de la estructura metodológica que permite realizar una integración significativa es la operacionalización del concepto que se busca medir. Esto es crucial para llevar a cabo integrar, ya que al desagregar un concepto en dimensiones - y estas a su vez en indicadores_-, se plantea una idea clara de lo que se necesita saber para medir tal concepto. De esta manera, si se recaba la información indispensable de manera cualitativa, es posible procesarla mediante la integración metodológica para tener resultados cuantitativos medibles que puedan probar la hipótesis.

En tal sentido, el instrumento que se plantea - al necesitar procesar datos de manera cualitativa y cuantitativa - puede ser desarrollado con la ayuda de softwares. Uno de los más útiles para este instrumento es Atlas ti. Este ayuda a organizar, interpretar y codificar la información recabada creando códigos o categorías, de manera que se pueda agrupar en temas (que pueden ser los indicadores, las dimensiones o los temas que se buscan probar). De esta manera se pueden conseguir argumentos claros por tema. Otro programa útil es Microsoft Office Excel, el cual se puede emplear para calcular las cifras que resulten de la codificación cuantitativa.

\section{Resultados}

\section{Un ejemplo de aplicación de la integración metodológica en estudios de caso comparativos}




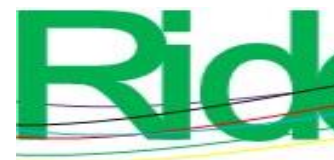

Revista Iberoamericana para la Investigación y el Desarrollo Educativo ISSN 2007 - 7467

Para ejemplificar una forma de aplicar la integración metodológica en el estudio de fenómenos de las RR. II., se presentan los resultados de una investigación titulada Participación ciudadana juvenil en Baja California (México) y California (Estados Unidos): el caso de los Modelos de Naciones Unidas (Márquez Duarte, 2018).

Para medir la participación ciudadana juvenil se otorgó un mayor valor a los siguientes indicadores: en la dimensión social se le otorgó el doble de valor (x2) a participar en organizaciones de la sociedad civil, y en la dimensión electoral-política se le dio el doble de valor (x2) a votar en elecciones, y el triple (x3) a ser candidato (tabla 2). Esta decisión se tomó con base en la bibliografía analizada, ya que varios autores aseveran que el voto, así como el ser candidato tiene mayores implicaciones políticas para los individuos, al menos en el contexto de México y América Latina. Por eso, se decidió que participar en OSC tenía un mayor peso que participar en los otros indicadores de la dimensión social, ya que implica mayor compromiso; además, las acciones y proyectos de las OCS generalmente tienen mayores implicaciones que las de las otras organizaciones contempladas como indicadores de la dimensión social. Con esta mayor ponderación se intentó conseguir un resultado de la medición de la participación ciudadana juvenil más ajustado a la realidad de los contextos para ubicar de manera más exacta a los individuos en las escalas utilizadas. 
Tabla 2. Operacionalización de la participación ciudadana juvenil

\begin{tabular}{|c|c|c|}
\hline Dimensiones & Indicadores & Medición \\
\hline Social & $\begin{array}{l}\text { 1. Participar en OCS (x2) } \\
\text { 2. Participar en } \\
\text { voluntariado } \\
\text { 3. Participar en comités } \\
\text { vecinales } \\
\text { 4. Participar en grupos } \\
\text { comunitarios }\end{array}$ & $\begin{array}{l}0 \text { a } 3 \text { escala de Likert- } \\
\text { Thurstone } \\
0=\text { no participa } \\
1=\text { participación sin toma } \\
\text { de decisiones } \\
2=\text { es parte en la } \\
\text { discusión de decisiones, } \\
\text { pero no las toma, puede } \\
\text { tener un puesto en la } \\
\text { organización } \\
3=\text { puesto directivo, toma } \\
\text { decisiones, dirige } \\
\text { proyectos }\end{array}$ \\
\hline $\begin{array}{l}\text { Directa (en mecanismos } \\
\text { de democracia directa) }\end{array}$ & $\begin{array}{l}\text { 1. Iniciativa de ley } \\
\text { 2. Referéndum } \\
\text { 3. Plebiscito } \\
\text { 4. Consultas ciudadanas } \\
\text { 5. Asambleas ciudadanas } \\
\text { 6. Talleres participativos } \\
\text { 7. Marchas } \\
\text { 8. Protestas }\end{array}$ & $\begin{array}{l}\text { Escala dicotómica } \\
0=\text { no participa } \\
1 \text { = sí participa }\end{array}$ \\
\hline Electoral-política & $\begin{array}{l}\text { 1. Votar en elecciones } \\
\text { (x2) } \\
\text { 2. Ser candidato (x3) } \\
\text { 3. Participar en partidos } \\
\text { políticos } \\
\text { 4. Participar en campañas } \\
\text { políticas } \\
\text { 5. Cabildeo con políticos } \\
\text { 6. Publicar en medios } \\
\text { sobre política/políticos }\end{array}$ & $\begin{array}{l}\begin{array}{l}1,2,5 \text { y } 6 \text { escala } \\
\text { dicotómica }\end{array} \\
0=\text { no participa } \\
1=\text { sí participa } \\
3 \text { y } 4 \text { = escala Likert- } \\
\text { Thurstone ( } 0 \text { a } 3) \\
0=\text { no participa } \\
1=\text { participación sin toma } \\
\text { de decisiones } \\
2=\text { toma parte en la } \\
\text { discusión de decisiones, } \\
\text { pero no las toma, puede } \\
\text { tener un puesto en la } \\
\text { organización } \\
3=\text { puesto directivo, toma } \\
\text { decisiones, dirige } \\
\text { proyectos }\end{array}$ \\
\hline
\end{tabular}

Fuente: Elaboración propia 


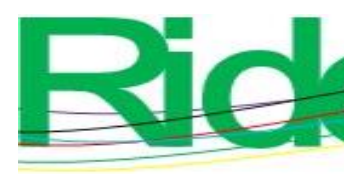

Revista Iberoamericana para la Investigación y el Desarrollo Educativo ISSN $2007-7467$

Al tener definida la forma de ponderación, así como la información recabada, se procedió a realizar los análisis y cálculos necesarios para obtener los puntajes de cada dimensión y los totales del cambio de participación ciudadana juvenil de cada uno de los MUN entrevistados, así como para ubicarlos por resultado en las dos escalas utilizadas para la interpretación de estos datos. Teniendo los resultados del cambio de la participación ciudadana juvenil por dimensión de cada MUN entrevistado, se ubica a cada uno de ellos en la escalera de Hart (2013) (para las dimensiones social y electoralpolítica) y en el espectro de participación pública (para la dimensión directa).

Hart (1993) declara que de los escalones 1 al 3, las acciones de los jóvenes se consideran "no participativas"; en cambio, de los escalones 4 al 8, Hart señala que se pueden categorizar como "participación genuina de los jóvenes". Para efectos de esta investigación, la siguiente es la forma de ubicar a los MUN entrevistados en esta escalera: para situarlos en los escalones del 1 al 3, pueden tener como máximo un 1 de resultado en cada indicador de la dimensión social, en la dimensión electoral-política pueden tener máximo un 1 en cada indicador, así como no tienen que haber realizado lobbying o publicado en medios sobre política o políticos, ya que al realizar alguna de estas acciones están iniciando formas de participación por motivación propia, con entendimiento de la situación. Depende del caso de cada individuo se evalúa si se ubica en el cuarto escalón, ya que los puntajes que debería tener por indicador son los mismos que para los escalones anteriores. Para ubicarlos en los escalones 5 o 6 los MUN entrevistados tienen que tener máximo un 2 como resultado de cada indicador de ambas dimensiones, incluyendo el realizar lobbying o publicar sobre política/políticos. Finalmente, para que los jóvenes puedan ubicarse en el escalón 7 u 8, tienen que tener 3 en cualquier indicador y se analiza la situación específica de cada individuo entrevistado.

Los resultados que se obtuvieron por medio de la integración metodológica fueron los siguientes: en 16 de los 18 entrevistados hay un aumento en la participación ciudadana juvenil derivado de su involucramiento en los MUN. En cuanto a la participación ciudadana juvenil total, hay más aumento que en la participación por cada una de las dimensiones, ya que, al sumarse el cambio en las tres dimensiones, 16 MUN tienen como resultado una cifra positiva. Esto quiere decir que $88.8 \%$ de los entrevistados aseguran que los MUN fomentaron su participación ciudadana juvenil. Además, el promedio del cambio de la participación ciudadana juvenil de los entrevistados fue de 6.94, lo que indica que no fue un cambio mínimo, sino significativo (Márquez Duarte, 2018). Este resultado constata que el aumento de la participación ciudadana juvenil causada por los MUN es significativo. En cuanto a la diferencia entre MUN mexicanos y 
estadounidenses, en la tabla 3 se aprecia que el aumento de la participación ciudadana juvenil fue mayor en MUN estadounidenses por dimensión y total.

Tabla 3. Cambio de participación ciudadana juvenil fomentado por los MUN en entrevistados mexicanos y estadounidenses (por dimensión y total)

\begin{tabular}{|l|l|l|}
\hline Tipo de dimensión & $\begin{array}{l}\text { Promedio de MUN } \\
\text { mexicanos }\end{array}$ & $\begin{array}{l}\text { Promedio de MUN } \\
\text { estadounidenses }\end{array}$ \\
\hline Dimensión social & 3 & 4.3 \\
\hline Dimensión directa & 1.4 & 2.3 \\
\hline $\begin{array}{l}\text { Dimensión electoral- } \\
\text { política }\end{array}$ & 0.6 & 3.6 \\
\hline $\begin{array}{l}\text { Participación ciudadana } \\
\text { juvenil total }\end{array}$ & 4.9 & 10.1 \\
\hline
\end{tabular}

Fuente: Elaboración propia

Por otra parte, se percibe que la dimensión directa de la participación ciudadana juvenil es la menos fomentada por los MUN, donde solo 11 de los 18 entrevistados refieren un cambio, específicamente un aumento. En cuanto a la dimensión electoralpolítica, 12 entrevistados indican un aumento, 4 señalan una disminución y dos no refieren cambios. Si analizamos el total del cambio de la participación ciudadana juvenil, se percibe que 16 de los 18 entrevistados señalan un aumento y solo 2 entrevistados refieren una disminución de la participación ciudadana juvenil. En cuanto a la participación ciudadana juvenil total, hay más aumento que en la participación por cada una de las dimensiones, ya que al sumarse el cambio en las tres dimensiones, 16 MUN tienen como resultado una cifra positiva.

\section{Discusión}

En este punto es importante aclarar que las conclusiones a las que se llegó se basan en la información cuantitativa y cualitativa analizada mediante el método comparativo y la integración metodológica, ya que estas formas de procesamiento se complementan y pueden clarificar la información para llegar a una conclusión más integral. En tal sentido, la articulación de estos enfoques metodológicos de las RR. II. y la discusión conceptual de participación ciudadana juvenil, MUN y formación ciudadana funcionaron como soporte de la investigación, y han ampliado los hallazgos del trabajo de campo, los cuales han permitido probar las hipótesis y aportar al conocimiento generado, ya que apoyan postulados de investigaciones anteriores que han servido como contexto. En síntesis, las 
capacidades fomentadas por los MUN en su totalidad fueron 19: presencia ejecutiva, organización, disciplina, debate, lenguaje formal, redacción de documentos formales, expresión oral, investigación/ser analítico, negociación, liderazgo, trabajo en equipo, cross-cultural understanding, seguir reglas, autoconfianza, networking, practicar idiomas, trabajo bajo presión, toma de decisiones, y tolerancia y empatía.

\section{Conclusiones}

Las RR. II., como ciencia dinámica, han enfrentado diversos debates paradigmáticos desde su creación derivados de la evolución de su objeto de estudio, lo que ha resultado determinante para la creación y adaptación de metodologías capaces de responder a la cambiante realidad internacional de una manera ágil, pues un movimiento en la agenda global puede modificar la funcionalidad de los paradigmas y sus metodologías casi de manera inmediata. Por eso, en este artículo se planteó la necesidad de emplear distintas metodologías para adaptarlas a los temas de la ciencia de las RR. II., con lo cual se procuró dar fortaleza a los estudios internacionales e ir dotando a la ciencia de diferentes opciones de análisis.

En este sentido, se exploró el método comparativo, el cual permitió explicar patrones y regularidades entre distintos sistemas sociales, políticos e institucionales, así como identificar posibles variables independientes que causan e influencian las variables dependientes. Con este método un desarrollo teórico y de construcción de hipótesis resulta fundamental, así como la construcción de la operacionalización.

En el caso de la integración metodológica, es un instrumento muy útil para las técnicas de investigación de corte mixto. Dentro de la $\mathrm{CP}$ y las RR. II., aporta herramientas relevantes, sobre todo para los trabajos de estudio de caso que se complementan perfectamente con la técnica de entrevistas con actores e informantes clave. En este sentido, el aporte principal de esta metodología es la medición de algún aspecto o conjunto de aspectos. Una parte crucial de la estructura metodológica que permite realizar una integración significativa es la operacionalización del concepto que se busca medir. Una correcta estructuración del concepto estudiado es crucial para integrar y para obtener de manera clara el conjunto de variables e indicadores a medir. De esta manera, si se recaba la información necesaria de manera cualitativa, es posible procesarla con el apoyo de los softwares informáticos para probar la hipótesis.

Como se analizó en el ejemplo planteado, la metodología de integración metodológica, el método comparativo y el estudio de caso son complementarios dentro de la ciencia de las RR. II. Si bien en este artículo se analizaron casos que poseen muchas 
especificidades en el ámbito regional — Baja California (México) y California (Estados Unidos)—, también pueden utilizarse en unidades macrosociales, como por ejemplo en el estudio comparativo de los Estados nación.

En el caso de esta investigación, al aplicar dichas metodologías para el entendimiento de fenómenos que corresponden a la agenda internacional, se pudo constatar que son útiles para medir un conjunto de datos que poseen una temporalidad transversal en donde se comparan similitudes y diferencias en dos áreas geográficas muy distintas; aun así, esto se pudo lograr únicamente con una conjunción de métodos cualitativos y cuantitativos, lo que permitió la comprobación del supuesto principal de esta investigación, es decir, que los MUN entrevistados refieren cambios en su forma de pensar y actuar en asuntos públicos, capacidades referidas por los entrevistados como conciencia política, liderazgo, autoconfianza y ser analítico. Esto los ha llevado a involucrarse activamente en los asuntos públicos y a lograr que su voz sea escuchada en los procesos de toma de decisiones públicas.

\section{Futuras líneas de investigación}

Como futura línea de investigación es importante seguir el estudio de las metodologías de corte cualitativo y cuantitativo para lograr enriquecer la ciencia de las RR. II., pues - como se mencionó a lo largo de este trabajo - durante muchos años las teorías se basaban en inferencias y en indicadores abstractos que eran imposibles de medir, como la naturaleza humana o el análisis del comportamiento de individuos específicos. Con los instrumentos metodológicos aquí planteados se ha logrado responder a estas críticas, dotando a la ciencia de las RR .II. de mayor credibilidad y legitimidad. 


\section{Referencias}

Bozal, M. G. (2006). Escala mixta Likert-Thurstone. Anduli, Revista Andaluza de Ciencias Sociales, 0(5), 81-95.

Centro de Información de la ONU para México, Cuba y República Dominicana [CINU] (s. f.). ¿Qué es un Modelo de Naciones Unidas? México. Recuperado de https://www.cinu.mx/modelos/preguntas-frecuentes/

Collier, D. (2011). Understanding process tracing. PS: Political Science \& Politics, 44(4), 823-830.

Creswell, J. W. (2003). Research design: Qualitative, quantitative, and mixed methods approaches (vol. 4). Sage Thousand Oaks, CA.

Gomm, R., Hammersley, M. and Foster, P. (eds.) (2000). Case Study Method: Key Issues, Key Texts. SAGE.

Hamui, A. y Varela, M. (2013). La técnica de grupos focales. Investigación en Educación Médica, 2(5), 55-60. Recuperado de http://www.scielo.org.mx/scielo.php?pid=S2007-

50572013000100009\&script=sci_abstract

Hart, R. A. (1993). La participación de los niños: de la participación simbólica a la participación autentica, Innocenti Essay no. 4, International Child Development Centre, Florence

Hart, R. A. (2013). Children's participation: The theory and practice of involving young citizens in community development and environmental care. Esarthscan. Retrieved from https://books.google.com/books?hl=es\&lr=\&id=ncRSAQAAQBAJ\&oi=fnd\&pg $=\mathrm{PP} 1 \& \mathrm{dq}=$ children $\% 27 \mathrm{~s}+$ participation+ladder+hart\&ots $=\mathrm{u} 5 \mathrm{lusP} 2 \mathrm{eFB} \& \mathrm{sig}=7 \mathrm{y} 7$ LbF6rZ6BMIHTmfj9jyKtlDmo

Hernández-Sampieri, R., Fernández, C. y Baptista, P. (2010). Metodología de la Investigación (20.a ed.). Mc Graw Hill.

Hurtado, J. (2016). La etnografía como método en las relaciones internacionales: una aproximación desde los estudios de la seguridad. El Internacionalista. Recuperado de https://revistaceimexico.blogspot.com/2016/09/la-etnografia-como-metodoen-las.html

Klotz, A. and Prakash, D. (2008). Qualitative methods in international relations. Palgrave Macmillan.

Lamont, C. (2015). Research methods in international relations. Sage. 
Lijphart, A. (1971). Comparative politics and the comparative method. The American Political Science Review, 65(3), 682-693. Doi: https://doi.org/10.2307/1955513

Lorenzo Domínguez, E. y Xalabarder Aulet, M. (1997). La investigación científica a través de internet: el ejemplo de los movimientos migratorios en el mundo. Ar@cne: Revista Electrónica de Recursos en Internet sobre Geografía y Ciencias Sociales, $1-9$. Recuperado de https://www.raco.cat/index.php/Aracne/article/view/64255

Márquez Duarte, F. D. (2018). Participación ciudadana juvenil en Baja California, México y California, Estados Unidos: el caso de los Modelos de Naciones Unidas (tesis de maestría). México: El Colegio de la Frontera Norte.

Monje, C. (2011). Metodología de la investigación cuantitativa y cualitativa. Guía didáctica. Universidad Surcolombiana.

Nohlen, D., Schultze, R. y Romano, M. (2006). Diccionario de ciencia política: teorías, métodos, conceptos (vol. 1, 2). México: Editorial Porrúa-El Colegio de Veracruz.

Obendorf, S. and Randerson, C. (2012). The Model United Nations simulation and the student as producer agenda. Enhancing Learning in the Social Sciences, 4(3). Retrieved from http://www.tandfonline.com/doi/full/10.11120/elss.2012.04030007

Patterson, J. R. (1996). Model United Nations simulations: An inquiry into active learning, role-playing, and role identification as they impact participants' sense of political efficacy [Ph.D.]. USA: Ann Arbor. Retrieved from http://ras.colef.mx:2186/pqdtglobal/docview/304246109/abstract/5430BC041D7

\section{$\mathrm{A} 4 \mathrm{~B} 08 \mathrm{PQ} / 4$}

Rojas, D. (2004). La historia y las relaciones internacionales: de la historia inter-nacional a la historia global. Historia Crítica, (27). Recuperado de https://www.redalyc.org/articulo.oa?id=81102709

Salazar, G. (2004). Las fuentes de la investigación en las relaciones internacionales. Revista CIDOB D’afers Internacionals, (64). Recuperado de https://www.cidob.org/es/articulos/revista_cidob_d_afers_internacionals/las_fue ntes_de_la_investigacion_en_las_relaciones_internacionales

Savolainen, J. (1994). The rationality of drawing big conclusions based on small samples: In defense of Mill's methods. Social forces, 72(4), 1217-1224.

Stuart, G. (2017). What is the Spectrum of Public Participation? Sustaining Community. Retrieved from 
https://sustainingcommunity.wordpress.com/2017/02/14/spectrum-of-publicparticipation/

Zaks, S. (2017). Relationships Among Rivals (RAR): A Framework for Analyzing Contending Hypotheses in Process Tracing. Political Analysis, 25(3), 344-362.

Zenuk-Nishide, L. (2014). Rationale and Theoretical Foundation for a Model United Nations Class. Journal of Foreign Studies, 64(2), 33-51.

\begin{tabular}{|c|c|}
\hline Rol de Contribución & Autor (es) \\
\hline Conceptualización & Kenia María Ramírez Meda \\
\hline Metodología & Fernando David Márquez Duarte \\
\hline Software & Fernando David Márquez Duarte \\
\hline Validación & Fernando David Márquez Duarte \\
\hline Análisis Formal & Fernando David Márquez Duarte \\
\hline Investigación & Kenia María Ramírez Meda. \\
\hline Recursos & $\begin{array}{l}\text { Kenia María Ramírez Meda (principal) /Fernando David } \\
\text { Márquez Duarte. }\end{array}$ \\
\hline Curación de datos & Fernando David Márquez Duarte \\
\hline $\begin{array}{l}\text { Escritura - Preparación del } \\
\text { borrador original }\end{array}$ & Kenia María Ramírez Meda \\
\hline $\begin{array}{l}\text { Escritura - Revisión y } \\
\text { edición }\end{array}$ & Kenia María Ramírez Meda \\
\hline Visualización & Kenia María Ramírez Meda \\
\hline Supervisión & $\begin{array}{l}\text { Kenia María Ramírez Meda (principal) /Fernando David } \\
\text { Márquez Duarte. }\end{array}$ \\
\hline Administración de Proyectos & Fernando David Márquez Duarte \\
\hline Adquisición de fondos & $\begin{array}{l}\text { Fernando David Márquez Duarte (principal) Kenia María } \\
\text { Ramírez Meda }\end{array}$ \\
\hline
\end{tabular}

